\section{P3-S2.05 INVASIVE OBSTETRIC PROCEDURES AND CESAREAN SECTIONS IN WOMEN WITH KNOWN HERPES SIMPLEX VIRUS (HSV) STATUS DURING PREGNANCY}

doi:10.1136/sextrans-2011-050108.449

N Moss, C Gardella, L Drolette, A Magaret, A Wald. University of Washington, Seattle, USA

Background Breach of neonatal skin by obstetric procedures during delivery increases risk of neonatal herpes among infants of women with genital HSV infections. We hypothesised that knowledge of genital HSV infection by clinical history or antibody tests (GH/ HSV-2) prior to delivery would result in reduced use of invasive obstetric procedures, and we explored whether use of invasive procedures in infected women on suppressive antiviral therapy was similar to use in women without infection.

Methods We reviewed 750 consecutive deliveries at an academic medical center in 2006; routine prenatal testing included HSV-1 and HSV-2 serostatus. The primary outcome was a composite of invasive delivery procedures (fetal scalp electrodes, artificial rupture of membranes, intrauterine pressure catheter, vacuum extraction or forceps extraction) among women with vs without GH/HSV-2. Women with planned c-sections, multiple gestation pregnancy, or genital herpes lesions at term were excluded.

Results 453 women, including 35 with a history of genital herpes (24 with HSV-2, 11 with HSV-1) and 59 with HSV-2 antibody but no history of genital herpes, were included in the analysis. Of the 94 women with GH/HSV-2, 56 (59.6\%) received suppressive antiviral therapy. Among the 38 women with GH/HSV-2 not on suppressive therapy, 15 (39.5\%) had an invasive obstetric procedure at delivery compared with 204 (57\%) of 359 women without GH/HSV-2 $(\mathrm{OR}=0.50 ; 95 \% \mathrm{CI}: 0.25$ to 0.98$)$. In contrast, no difference in unplanned c-sections was observed by GH/HSV-2 status (29\% vs $28 \%$ ). Among the 56 women with GH/HSV-2 treated with antiviral therapy, the risk of invasive procedures was similar to that of women without $\mathrm{GH} / \mathrm{HSV}-2,62.5 \%$ vs $57 \%(\mathrm{OR}=1.27 ; 95 \% \mathrm{CI} 0.71$ to 2.26).

Conclusion Women with known genital herpes or HSV-2 positivity are less likely to undergo invasive procedures that increase the risk of neonatal herpes. However, obstetricians do not appear to avoid these procedures in women who are on suppressive therapy. These data suggest physicians who know their patients' HSV-2 status in pregnancy alter their behaviour to maintain the integrity of neonatal skin. Given the frequency of breakthrough HSV-2 shedding on suppressive antiviral therapy, and the occurrence of neonatal herpes in infants born to women on suppressive therapy, the high frequency of invasive procedure use among women on antiviral therapy is concerning.

\section{Clinical sciences poster session 3: HIV P3-S3.01 ANAL PAP SMEAR SCREENING AMONG DRUG USERS LIVING WITH HIV IN MIAMI, FLORIDA}

doi:10.1136/sextrans-2011-050108.450

I Rosa-Cunha, T Hooton, G Cardenas, L Metsch. University of Miami, Miami, USA

Background Human papillomavirus (HPV) is associated with anal cancer. Human immunodeficiency virus (HIV) infection and cocaine use are associated with increased risk for HPV infection and associated diseases, but little is known about anal dysplasia among HIVinfected drug users. The objective of our study was to assess the rate of abnormal anal Pap smears among drug users living with HIV in Miami, Florida.

Methods Project HOPE (Hospital is an Opportunity for Prevention and Engagement) is a two-site study conducted in Miami and
Atlanta to evaluate the efficacy of a brief prevention intervention for HIV-positive crack cocaine users recruited from two inner-city hospitals during their inpatient stays. At the Miami site, anal Pap smears from women and men $(n=46)$ were collected.

Results The study population was $95 \%$ Black, the mean age was 47 , $63 \%$ were female (29/46), 35\% heterosexual males (16/46) and $2 \%$ men who have sex with men (MSM) (1/46). Approximately half of the participants did not complete high school, $50 \%$ were on antiretroviral therapy and $50 \%$ had been diagnosed with HIV for more than 14 years. The median CD4 cell count was 198. Overall 69.5\% $(32 / 46)$ of anal Pap smears were abnormal; 69\% of women $(20 / 29)$ and $70.5 \%$ of men $(12 / 17)$ had abnormal Pap smear.

Conclusions These preliminary data suggest that abnormal anal Pap smears are common in drug users living with HIV in Miami and highlight the need for further studies on prevention and screening of anal dysplasia and cancer in this population.

\section{P3-S3.02 REITER'S SYNDROME IN ASSOCIATION WITH HIV INFECTION: REPORT OF THREE CASES}

doi:10.1136/sextrans-2011-050108.451

A Inamadar. BLDE University, Bijapur, India

Introduction Reiter's syndrome is a relatively rare, non-suppurative, sero-negative arthropathy seen among young adults with HLAB27. Epidemic (post-enteric) and endemic (sexually acquired) variants of the disease may occur. Reiter's disease is difficult to manage because of its chronic recurring course. The prevalence of Reiter's syndrome in HIVinfected patients varies between $1.7 \%$ and $11.2 \%$. The course of the disease is more severe and progressive in these patients and often refractory to treatment. Here, clinical features and management of three HIV infected patients with Reiter's syndrome have been described.

Case reports Three heterosexual men, age ranging from 20 to 40 years presented with psoriasiform skin lesions, dystrophic nail changes and incapacitating arthritis involving bilateral knees, ankles and toes of 3-6 months duration. Two of the patients had asymptomatic balanitis. Skin lesions, keratoderma blenorrhagica, were generalised and severe in one patient and were restricted to extremities in two others. There was no history of preceding gastro-intestinal infection or urethritis in any of them. All the three patients had history of unprotected sex with commercial sex workers in the past. They were tested HIV positive (Western blot method) and CD4 T cell count was 550, 489 and 450 respectively. The patients were treated with a combination of indomethacin, sulfasalazine for the arthritis and systemic isotretinoin. Arthritis responded to sulfasalazine and indomethacin. Cutaneous and nail changes responded to systemic isotretinoin. Long term treatment was required for all patients and one had recurrent flare-up of symptoms.

Conclusion Reiter's syndrome is one of the causes of rheumatic disorders in HIV-infected patients. Direct etiological role of HIV infection in the development of Reiter's syndrome is not established. Reiter's syndrome as a manifestation of immune reconstitution syndrome in a HIV-infected patient has been reported. Clinical severity of the disease may be increased because of underlying immunosuppression. These patients present with therapeutic challenge because of the restricted opportunity to use conventional immunosuppressive therapeutic agents.

\section{P3-S3.03 CLINICAL PROFILE OF BUSCHKE-LOWENSTEIN TUMOUR IN HIV INFECTED PATIENTS}

doi:10.1136/sextrans-2011-050108.452

R Shivanna. BLDE University, Bijapur, India

Introduction Buschke Löwenstein tumour (BLT) is a rare Human Pappiloma Virus (HPV)- triggered indolent lesion involving perineal 
and/or perianal skin. The lesions are locally invasive and difficult to manage because of the voluminous tissue bulk and recurrence potential. Underlying immune defect in the background of HIV infection may enhance tumour-aggressiveness in these lesions. Three HIV-infected patients with BLT have been reported.

Case reports Patient 1: A 46-year-old woman presented with a cauliflower-like pink, fleshy mass of 18 months duration, protruding through the anal orifice, causing discomfort and disturbance in defecation. She was HIV-infected, acquired through conjugal relation. In addition to the above lesion, she had multiple genital molluscum contagiosum and bowenoid papulosis. Her CD4 T cell count was 350 and she was not on antiretroviral therapy (ART). Histopathology of the lesion was suggestive of BLT. The patient was treated with cryotherapy followed by surgical debulking of the lesion. ART was advised. Patient 2: A 35-year-old commercial sex worker presented with a large, variegated growth involving labia minora, extending to perineum, perianal region and lower part of gluteal folds, since last 5 years. There was sudden increase in extent and ulceration of the lesion since last 6 months. In the pubic region she had a conglomerated lesion of multiple condyloma acuminata and multiple bowenoid papulosis. She was HIV-infected with CD4 T cell count of 660. The lesion was biopsied from multiple sites and histopathological examination was consistent with BLTwith grade II squamous cell carcinoma in some specimens. The patient was treated with surgical debulking followed by radiotherapy. Patient 3: A 40-year-old man with history of high risk sexual practices and intravenous drug abuse presented with multiple, fleshy masses over penis, scrotum and peno-scrotal junction, giving rise to deformed appearance of the genitalia. The lesions were present since several years with slow growth. He was HIV positive with a CD4 T cell count of 300 . He was on ART since last 6 months. Histopathology of the lesion was suggestive of BLT. The patient was treated with cryotherapy followed by surgery.

Conclusion HIV-infected patients with Buschke Löwenstein tumour are at a higher risk of developing invasive squamous cell carcinoma because of higher incidence of abnormal anal and genital cytology, presence of HPV-DNA in these cells and a low CD4 T cell count.

\section{P3-S3.04 CLINICAL SCREENING OF SEXUALLY TRANSMITTED INFECTIONS AND RISK FACTORS AMING PEOPLE LIVING WITH HIV AT THE UNIVERSITY HOSPITAL OF COTONOU}

doi:10.1136/sextrans-2011-050108.453

${ }^{1} \mathrm{C}$ Ahouada, ${ }^{1} \mathrm{~J}$ Bashi, ${ }^{2} \mathrm{M}$ D Zannou, ${ }^{1} \mathrm{~A}$ Gougounon, ${ }^{1} \mathrm{~J}$ Akakpo, ${ }^{1} \mathrm{R}$ Zinsou, ${ }^{1} \mathrm{C}$ d'Almeida, ${ }^{1} \mathrm{C}$ Ahomadegbe, ${ }^{2} \mathrm{~A}$ Azon-kouanou, ${ }^{2} \mathrm{G}$ Ade, ${ }^{2} \mathrm{~F}$ Houngbe. ${ }^{1}$ Amulatory treatment center of academyc hospital, cotonou, Benin; ${ }^{2}$ Department of internal medicine, Benin

Background Many studies have shown an association between the presence of a sexually transmitted infection (STI) and the occurrence of HIV infection. Because of improved prognosis and quality of life due to antiretroviral (ARV), people living with HIV (PLHIV) tend to abandon safer sex practices. The consequence could be the resurgence of sexually transmitted infections. The objective of this study is to determine the prevalence of STIs and associated factors in the population of PLHIV in order to lay appropriate prevention strategies.

Methods This is a descriptive cross-sectional study conducted from January to February 2011 on PLHIV admitted in consultation at Centre de Traitement ambulatoire, of academic Hospital of Cotonou. An interrogation and urogenital examination were realised systematically by physicians in all patients. STI diagnosis was presumptive, based on observed lesions. The dependent variable studied is the presence of suggestive lesions of STIs. The data entry was made using Epi Data 3.1 and statistical analysis with SAS version 9.2. Proportions were compared using the $\chi^{2}$ test. The significance level was $5 \%$ and CIs at $95 \%$.

Results 85 patients were recruited, 27 (31.76\%) had suggestive lesions of STIs. The STIs lesions found in genitals were: irritation $(21.18 \%)$, papular lesions $(12.94 \%)$, discharge $(10.59 \%)$, ulcers $(9.41 \%)$, and malodorous vaginal discharge $(4.71 \%)$. The presumptive diagnosis raised in front of these lesions were: genital candidiasis $(23.53 \%)$, genital herpes (8.24\%), condyloma (3.53\%), Chlamydia $(2.35 \%)$, syphilis $(1.18 \%)$. STI prevalence was $25 \%$ in patients on ARVs as against $52 \%$ among untreated $\mathrm{OR} 0.30$ [0.10 to 0.84$], \mathrm{p}=0.02$. The median CD4 count at diagnosis was 379 cells/ $\mu$ l. The majority of patients, that to say $58 \%$, continues to maintain unprotected sex. They were $24 \%$ reported having procreated after knowledge of their positive HIV status. This indicates a risk of sexual transmission of infections.

Conclusion STIs are frequent in people with HIV even in patients treated with ARVs. Risk behaviours of STIs and HIV transmission are particularly observed in patients whose clinical and immunological status become satisfying. It appears important to emphasise the observance of security measures in monitoring of patients with HIV, especially when their clinical and immunological status are being improved.

\section{P3-S3.05 COTRIMOXAZOLE RESISTANT RESPIRATORY OPPORTUNISTIC PATHOGENS IN HIV AND AIDS PATIENTS IN LAGOS, NIGERIA}

doi:10.1136/sextrans-2011-050108.454

N Idika, M Anidobe, D Onwujekwe, 0 Idigbe. Nigerian Institute of Medical Research, Lagos, Nigeria

Introduction Opportunistic infections, viral, bacterial, fungal and parasitic are the most common cause of morbidity and mortality in HIV patients. Of the different antibiotics used for bacterial respiratory tract infections, cotrimoxazole appears to be a drug of choice in most developing countries because of its very low cost. Studies in Ivory Coast showed that cotrimoxazole decreased hospitalisation in $50 \%$ of all HIV patients and decreased mortality by $50 \%$ when given to HIV positive TB patients. Recently Streptococus pneumoniae, Haemophilus influenzae, Moraxalla catarhalis have become increasingly resistant to antibiotics and the rates vary between countries. Aware of the public health importance, the drug resistance patterns on bacterial respiratory opportunistic pathogens from HIV patients in Lagos were studied for better management of HIV in Nigeria.

Methods 310 sputum samples were collected from HIV patients presenting with respiratory complaints at ART clinics in Lagos after due informed consent from the patients and processed in the laboratory within $4 \mathrm{~h}$ using standard microbiological methods.

Results $57.1 \%$ patients were females, $70.5 \%$ of the samples grew bacterial pathogens mainly Morascella catarrholis 25.1\%, Staphylococcus aureus 9.2\%, Streptococcus pneumoniae 8.3\%, Pseudomonas aeruginosa $7.3 \%$, coaggulase negative staphylococcus 5.3\%. Some Enterobactereciae were isolated and $86.8 \%$ of isolates were susceptible to ofloxacin, $80.2 \%$ to ciprofloxacin and $12.8 \%$ to cotrimoxazole.

Conclusion Pathogens isolated were susceptible to ofloxacin, ciprofloxacin but highly resistant to cotrimoxazole, an affordable and widely used drug in African countries. This poses a challenge to management of HIV in Nigeria. 